\title{
About the origin of European spelt (Triticum spelta L.): allelic differentiation of the HMW Glutenin B1-1 and A1-2 subunit genes
}

Received: 20 January 2003 / Accepted: 13 August 2003 / Published online: 16 October 2003

(C) Springer-Verlag 2003

\begin{abstract}
To investigate the origin of European spelt (Triticum spelta L., genome AABBDD) and its relation to bread wheat (Triticum aestivum L., AABBDD), we analysed an approximately $1-\mathrm{kb}$ sequence, including a part of the promoter and the coding region, of the highmolecular-weight (HMW) glutenin B1-1 and A1-2 subunit genes in 58 accessions of hexa- and tetraploid wheat from different geographical regions. Six Glu-B1-1 and five Glu-A1-2 alleles were identified based on 21 and 19 informative sites, respectively, which suggests a polyphyletic origin of the A- and B-genomes of hexaploid wheat. In both genes, a group of alleles clustered in a distinct, so-called beta subclade. High frequencies of alleles from the Glu-B1-1 and Glu-A1-2 beta subclades differentiated European spelt from Asian spelt and bread wheat. This indicates different origins of European and Asian spelt, and that European spelt does not derive from the hulled progenitors of bread wheat. The conjoint differentiation of alleles of the A- and B-genome in European spelt suggests the introgression of a tetraploid wheat into free-threshing hexaploid wheat as the origin of European spelt.
\end{abstract}

Electronic Supplementary Material Supplementary material is available in the online version of this article at http://dx.doi.org/10.1007/s00122-003-1441-7

\section{Introduction}

Cytogenetic and molecular evidence indicate that freethreshing hexaploid bread wheat (Triticum aestivum L.; syn. T. aestivum ssp. aestivum; AABBDD; $2 \mathrm{n}=6 x=42$ ) derived by few mutations from hulled hexaploid progenitors (Kerber and Rowland 1974) which originated by

Communicated by J. Dvorak

R. H. E. Blatter $(\bowtie) \cdot$ S. Jacomet · A. Schlumbaum

Institut für Prähistorische und Naturwissenschaftliche Archäologie, Universität Basel, Spalenring 145, 4055 Basel, Switzerland

e-mail: Robert.Blatter@unibas.ch

Tel.: +41-61-2010210

Fax: +41-61-2010235 hybridisations of tetraploid wheat, probably emmer [Triticum dicoccum (Schrank) Schübl.; syn. Triticum turgidum ssp. dicoccum; AABB; $2 \mathrm{n}=4 x=28]$, and the diploid goatgrass Aegilops squarrosa Coss. (syn. Aegilops tauschii; DD; 2n=2x=14) (Kihara 1944; McFadden and Sears 1946) in the Southern regions of the Caspian Sea (Jaaska 1980; Dvorák et al. 1998). In contrast, the origin of hulled hexaploid spelt (Triticum spelta L.; syn. T. aestivum ssp. spelta; genome AABBDD; $2 \mathrm{n}=6 x=42$ ) is less clear. European spelt could originate from a hybridisation of tetraploid wheat and Aegilops squarrosa, which only occurs in Asia (Zohary and Hopf 2000), by deriving either from the hulled progenitors of bread wheat (Andrews 1964) or from an independent hybridisation. Subsequently, spelt would have spread like many other crops from the Near East into Europe where it was a staple crop since the Bronze Age, particularly in the Northern Alpine region. This hypothesis of an Asian origin of European spelt is supported by the traditional spelt cultivation in Asia (Kuckuck and Schiemann 1957) and by archaeological evidence (Zohary and Hopf 2000). However, discrepancies in the archaeological record (Nesbitt and Samuel 1996) and genetic differences between European and Asian spelt (e.g., Jaaska 1978; Dvorák and Luo 2001) argue against European spelt originating from Asia. An alternative hypothesis, which could explain the evolution of European spelt outside Asia, is that spelt evolved after the emergence of hexaploid bread wheat by a hybridisation of tetraploid wheat and free-threshing hexaploid wheat (e.g., Flaksberger 1930). The spelt-like offspring of such a cross was confirmed experimentally (MacKey 1966; Ohtsuka 1998). This hypothesis of European spelt evolution by an introgression of tetraploid wheat into free-threshing hexaploid wheat could explain both, the little differentiation among the D-genome of different hexaploid wheats; which argues against European spelt deriving from an independent hybridisation of tetraploid wheat and $\mathrm{Ae}$. squarrosa (Dvorák et al. 1998), and the genetic differences on the A- and B-genomes between European spelt and bread wheat, which argue against European spelt 
deriving from the hulled ancestors of bread wheat (e.g., Liu and Tsunewaki 1991; Siedler et al. 1994). However, the origin of European spelt is still controversial (for a review see Salamini et al. 2002).

In a previous study we have analysed a 240-bp fragment of all six high-molecular-weight (HMW) glutenin subunit genes in historical spelt and bread wheat (Blatter et al. 2002). The Glu-B1-1, Glu-A1-2 and GluB1-2 alleles, in a historical and an extant landrace of Swiss spelt, differed from the alleles in historical and modern bread wheat. The Glu-B1-1 spelt allele, however, was closely related to a Glu-B1-1 allele of the so called Glu-B1-1 beta subclade, which was previously identified only in a minority of tetraploid wheat (Brown 1999). The HMW glutenin alleles of the D-genome, in contrast, did not differentiate between spelt and bread wheat (Blatter et al. 2002). Although only few accessions were analysed, these results support that spelt originated from an introgression of tetraploid wheat into free-threshing hexaploid wheat. We have therefore extended our previous study by sequencing an approximately $1-\mathrm{kb}$ fragment, including a part of the promoter and the coding region, of the Glu-B1-1 and Glu-A1-2 genes mainly in extant spelt from different geographic regions, but also in extant bread wheat and some tetraploid wheat. The aims were to analyse the allelic variation of Glu-B1-1 and Glu-A1-2 genes in different wheat, and to investigate the geographic and taxonomic distribution of these alleles for gaining further information on the origin of European spelt and its relation to bread wheat.

\section{Materials and methods}

\section{Plant materials}

Forty three accessions of $T$. spelta L., 12 accessions of $T$. aestivum L., and three accessions of Triticum durum Desf. from different geographic regions were obtained from IPK Gatersleben, D. T. spelta 'Oberkulmer' was provided by B. Keller (University Zürich, $\mathrm{CH})$. The spelt accessions assigned to European spelt consisted of T. spelta L. ssp. spelta collected in Switzerland, Germany, Spain, Italy, former Czechoslovakia, Poland, Ukraine and unspecified locations in Europe. Spelt from Switzerland and Germany, the 'Northern Alpine region', was analysed as a subgroup within European spelt. The Asian spelt consisted of T. spelta L. ssp. spelta collected in Iran and T. spelta L. ssp. kuckuckianum Gökg. from Tajikistan, Uzbekistan, Turkmenistan and unknown locations in Asia. All accessions are listed in detail as 'Electronic Supplementary Material' (ESM). The nomenclature of Triticum and Aegilops species was used according to Miller (1987).

\section{DNA extraction}

DNA was extracted from 2 to 3 grains or fresh leaves of each accession either with a Nucleon PhytoPure kit (Amersham, Buckinghamshire, UK), according to manufacturer's instructions, or with a CTAB based method (Blatter et al. 2002).
PCR, cloning and sequencing

Fragments of the HMW Glu-B1-1 and Glu-A1-2 genes were PCR amplified in a Mastercycler gradient (Eppendorf, Hamburg, D) in $50 \mu \mathrm{l}$ reactions containing approximately $15 \mathrm{ng}$ of DNA, $5 \mu \mathrm{l}$ of Taq buffer, $50 \mu \mathrm{M}$ each of dNTP, $0.3 \mu \mathrm{M}$ of each primer and 1.25 units of Taq DNA polymerase (Roche, Mannheim, D). The forward primers were gene specific (Glu-B1-1: 5'-AAATGGGCTTTAGGAGAGATGG-3'; Glu-A1-2: 5'-CGTCATAGCACACATAAATGTTGC- $3^{\prime}$, whereas the reverse primer was designed universal for all HMW glutenin genes (5'-GAAGCTTGGCCTGGATARTATG-3'). A 'touchdown' PCR approach was applied. PCR conditions were: (1) $3 \mathrm{~min}$ at $94^{\circ} \mathrm{C}$; (2) seven cycles of $1 \mathrm{~min}$ starting at $64^{\circ} \mathrm{C}$, reducing the temperature $-\Delta 1^{\circ} \mathrm{C}$ per cycle (i.e. 'touchdown' at $58^{\circ} \mathrm{C}$ ), $1 \mathrm{~min}$ at $74^{\circ} \mathrm{C}, 1 \mathrm{~min}$ at $94^{\circ} \mathrm{C}$; (3) 33 cycles of $1 \mathrm{~min}$ at $58^{\circ} \mathrm{C}, 1 \mathrm{~min}$ at $74^{\circ} \mathrm{C}$ and $1 \mathrm{~min}$ at $94^{\circ} \mathrm{C}$; (4) $1 \mathrm{~min}$ at $58^{\circ} \mathrm{C}$; and (5) $9 \mathrm{~min}$ at $74^{\circ} \mathrm{C}$. The PCR products of the expected size were cloned with the p-GEM-T Vector Kit (Promega, Madison, Wis.) according to manufacturers instruction. One clone per PCR product was analysed. Plasmids were purified with QIAGEN miniprep plasmid kit (QIAGEN, Hilden, D) and sequenced on a ABI Prism 310 Genetic Analyser (Perkin Elmer, Foster City, Calif.). Based on the obtained sequences, specific reverse primers were designed for the Glu-B1-1 fragment (5'-ATTTGTTGGAGTTGCTGCGAAG- $\left.3^{\prime}\right)$ and the Glu-A1-2 fragment (5'-CCCTGCTGAGGAGATATTACAC- $3^{\prime}$ ). These primers were used to verify the Glu-B1-1 alleles in six accessions (nos. 21, 26, 30, 44, 64 and 71 see ESM) and the Glu-A1-2 alleles in five accessions (nos. $23,33,50,90$ and 91). The amplification was performed in 100- $\mu 1$ reactions with the same PCR conditions as above. PCR products were purified with the QIAquick PCR purification kit (QIAGEN, Hilden, D) and directly sequenced by Microsynth $\mathrm{GmbH}$ (Balgach, $\mathrm{CH}$ ) with the primers used for amplification. All sequences were submitted to EMBL with the accession numbers AJ566642AJ566698 and AJ567923-AJ567980.

\section{Data analysis}

Each sequence was compared to the database using FastA in the Wisconsin package Version 10.0 (GCG, Madison, Wis.). All sequences were aligned using SeqLab in the Wisconsin package. Informative sites were identified with the tool 'Show Character Status ...' in PAUP 4.0b8 (Sinauer Associates, Sunderland, Mass.). Singular variations were subsequently treated as PCR or cloning artefacts and were excluded from further analysis. Using this approach, it is possible that authentic variations were rejected. However, whenever an accession was analysed twice, either by direct sequencing or by analysing clones of independent PCR products, all informative sites in Fig. 2 were confirmed and no additional variation was identified. Insertions or deletions (indels) were considered singular events, and were substituted each by a transversion for the following analyses. Pairwise genetic distances (PGD) were calculated in PAUP 4.0b8. Cladograms were constructed using the TCS software (Clement et al. 2000). Putative sites of recombination were identified with the program 'Recombination' in the DnaSP package vers. 3.52 (Rozas and Rozas 1999). A phylogenetic tree was constructed using the maximum parsimony (MP) method in PAUP 4.0b8 including the Glu-B1-1 and A1-2 alleles (starting from position -368 and -375 , respectively) and the published sequence fragments of Glu-A1-1 (EMBL accession: X13928), Glu-D1-1 (X12928), Glu-B1-2 (X61026) and Glu-D1-2 (X12929) from T. aestivum. Positions with gaps were excluded from the analysis except for the indels in the Glu-B1-1 and A1-2 alleles substituted by a transversion (see above). One hundred bootstrap replicates were performed to test the stability of the tree. The MP-tree was drawn by midpoint rooting since the Glu-1-1 and Glu-1-2 genes are paralogous. The coding and the non-coding region were analysed together. The subclade of the Glu-B1-1 clade, which included the Glu-B1-1 alleles of T. spelta 'Oberkulmer' (accession no.1, see ESM) and of T. dicoccum var. tragii (EMBL accession X98714), was previously named beta subclade (Allaby et 
al. 1999; Blatter et al. 2002). Analogous to the Glu-B1-1 clade, the subclade with the Glu-A1-2 allele of T. spelta 'Oberkulmer' was named the Glu-A1-2 beta subclade.

Deviating from the nomenclature of McIntosh et al. (1998), Roman numbers were given to the identified alleles because the partial nucleotide sequences could only be assigned to the corresponding sections of the complete genes.

Two approximately 1-kb Glu-B1-1 and Glu-A1-2 sequences (EMBL accession: X03042 and X13927) and 18 sequences of shorter fragment length $(240 \mathrm{bp}$ ) from the Glu-B1-1 (position -278 to -38 ) and Glu-A1-2 (position -280 to -39 ) promoter region (AJ132190, AJ131816, AJ399894, AJ399896, X03042, X13927, X98585, X98587, X98715, X98714, Y10953, Y10954, Y10965, Y10966) were included in the cladograms and in the calculation of the allele frequencies. Each sequence from the database was assigned to a Glu-B1-1 or Glu-A1-2 allele, or, if the 240-bp fragments did not contain enough allele-specific sites, to a group of alleles (B1-1I-IV).

\section{Results}

A PCR product of the HMW Glu-B1-1 and Glu-A1-2 region was amplified from all 58 wheat accessions, except for Glu-A1-2 from accession no. 46 (see ESM). Only partial sequences were obtained from accessions no. 10 (Glu-B1-1) and no. 67 (Glu-A1-2) because the polymerase switched during an early elongation step of the PCR amplification from the targeted HMW glutenin template to another HMW glutenin gene ('jumping PCR'). The fragments were identical to the allele Glu-B1-1VI (position -338 to 359 ) or Glu-A1-2I (position -5 to 422 ), and both fragments were completed with the missing section of the respective allele.

In total, six Glu-B1-1 and five Glu-A1-2 alleles were identified. All alleles were more similar to reference sequences of the target gene than to any other HMW glutenin gene, which shows the correct amplification of the targeted HMW glutenin genes. This was confirmed by a MP-tree of all six HMW glutenin genes of hexaploid wheat, where the Glu-B1-1 and the Glu-A1-2 alleles grouped in clearly separate clades (Fig. 1).

\section{Alleles of the HMW Glu-B1-1 subunit gene}

Six Glu-B1-1 alleles, named Glu-B1-1I to Glu-B1-1VI, were identified by 18 transitions, one transversion, and two indels (21 informative sites) (Fig. 2a). The maximum variation between two Glu-B1-1 alleles (B1-1III and B11VI) was 14 informative sites [pairwise genetic distance (PGD) 0.0157]. The alleles differed in length from 874 to $943 \mathrm{bp}$ because of two indels at the positions -509 to -456 and 95 to 109 . The insertion at position -509 to -456 is a tandem duplication including the so-called 'cereal box' (Anderson and Greene 1989). This duplication was found in all Glu-B1-1 alleles, except Glu-B1-1III and in none of the other HMW glutenin subunit genes. Ten informative sites were situated within the promoter region and 11 within the coding region (Fig. 2a). Seven variations in the coding region were non-synonymous (position 46, 113, 128, 173, 215, 226 and 259) and

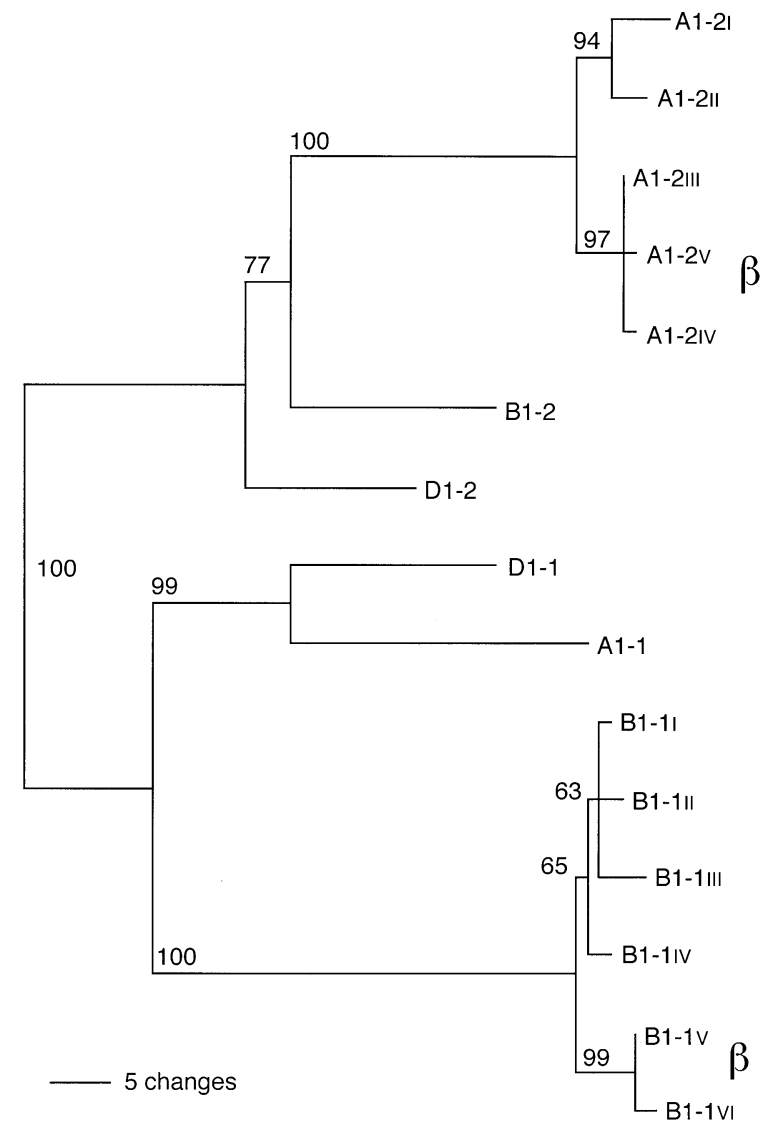

Fig. 1 A phylogenetic tree was calculated using the maximum parsimony (MP) method from an alignment of a 715-bp fragment of the Glu-B1-1 and Glu-A1-2 alleles found in this study, and of one representative allele each of the Glu-A1-1, Glu-D1-1, Glu-B1-2 and Glu-D1-2 genes from the database. The MP-tree was drawn by midpoint rooting with the Glu1-2 genes selected as an outgroup for the Glu1-1 genes. Bootstrap values were calculated from 100 replicate trees. All HMW glutenin subunit genes from the genomes A, B and D form clearly separate clades. The subclades of Glu-B11 and Glu-A1-2, which include the alleles from $T$. spelta 'Oberkulmer', were defined as beta subclades and are indicated with ' $\beta$ '

affected two cysteine residues (position 128 and 173) in the allele B1-1IV, which are responsible for the tertiary structure of the protein (Shewry et al. 1999).

The MP-tree of HMW glutenin genes from all genomes (Fig. 1), and the cladogram of the Glu-B1-1 alleles (Fig. 3a), revealed a split of the Glu-B1-1 clade into different subclades. One distinct subclade was formed by the alleles B1-1V and B1-1VI, which varied by 10 to 14 differences (PGD 0.0111-0.0157) from the other four alleles (B1-1I to B1-1IV). This corroborates previous results where the allele of $T$. spelta var. 'Oberkulmer' (B1-1VI) and an allele of tetraploid wheat (B1-1V) also clustered together in this so-called beta subclade (Blatter et al. 2002). The variation was two differences (PGD 0.0022) between the alleles of the beta subclade, and four to eight differences (PGD 0.0044 0.0089) among the non-beta alleles. A different mutation rate was indicated for the coding and the promoter region. 
Fig. 2 Informative sites are shown from six alleles of the HMW Glu-B1-1 subunit genes, named B1-1I to B1-1VI (a), and five alleles of the HMW GluA1-2 subunit genes, named A12I to A1-2V (b). Within the analysed 1-kb fragment the allele Glu-B1-1I corresponds to the allele Glu-B1-1b of T. aestivum cv 'Cheyenne', based on the official nomenclature of glutenin alleles (McIntosh et al. 1998). The allele Glu-A1-2II corresponds to the allele GluA1-2a of T. aestivum cv. "Chinese Spring'. Numbering of the nucleotide positions $(+1)$ starts at the ATG start codon. At positions with indels ' + ' marks the presence of an insertion, '-' marks the absence of an insertion. Reference sequences are from $T$. spelta var. arduinii (accession no. 3)
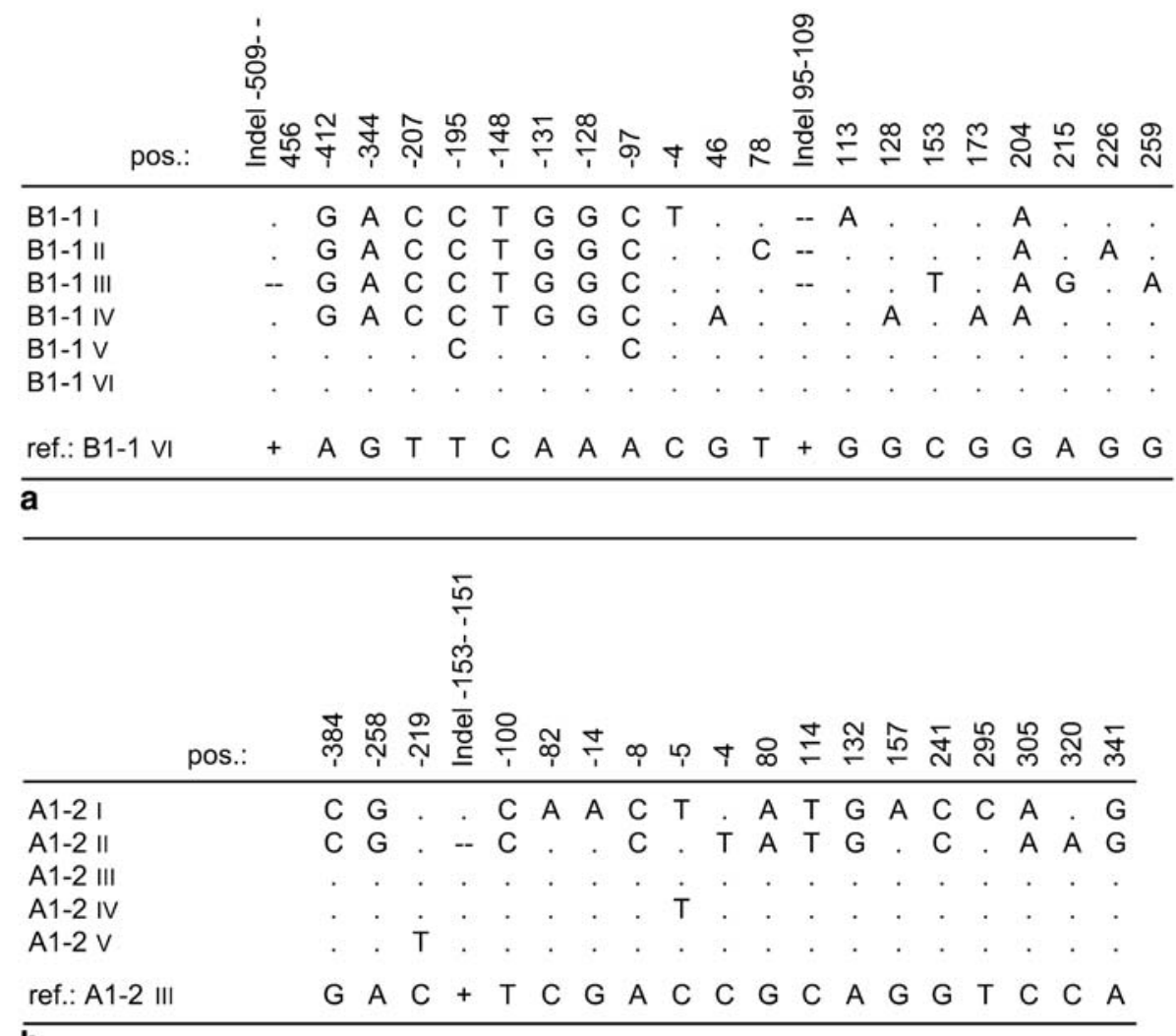

From seven informative sites separating the beta alleles from all other alleles, six were located in the promoter region and only one in the coding region. On the other hand, the alleles B1-1I to B1-1IV varied among each other by nine differences in the coding region, but only two differences in the promoter region (Figs. 2a and 3a).

\section{Alleles of the HMW Glu-A1-2 subunit gene}

Five Glu-A1-2 alleles, numbered Glu-A1-2I to Glu-A1$2 \mathrm{~V}$, were identified by 12 transitions, six transversions, and one indel (19 informative sites) (Fig. 2b). The maximum variation between two Glu-A1-2 alleles (A1-2I and $\mathrm{A} 1-2 \mathrm{~V}$ ) was 16 informative sites (PGD 0.0174). The alleles varied in length from 971 to 974 bp because of a missing triplet at position -153 to -151 in the promoter region of allele A1-2II. Ten informative sites were situated within the promoter region and nine within the coding region (Fig. 2b). Seven variations in the coding region were non-synonymous (positions 80, 157, 241, 295, 305, 320 and 341), but in contrast to Glu-B1-1, none affected the five cysteine residues, which are present in the N-terminal domains of all y-type HMW glutenin subunit genes (Shewry et al. 1999). The thymine instead of cytosine at position -5 in the alleles A1-2I and A1-2IV is either the result of homoplasy or of recombination events between the sites -8 to -5 and -5 to 80 .
The topology of the MP-tree of the HMW glutenin genes (Fig. 1) and of the cladogram of the Glu-A1-2 alleles (Fig. 3b) revealed a split of the Glu-A1-2 clade into different subclades, similar to the Glu-B1-1 clade. One subclade was formed by the alleles A1-2III to A1$2 \mathrm{~V}$, which varied from the alleles A1-2I and A1-2II by 13 to 16 differences (PGD 0.0142-0.0174). This subclade included the allele of $T$. spelta 'Oberkulmer' (A1-2V) and, in analogy to the Glu-B1-1 beta subclade, it was named the Glu-A1-2 beta subclade. The variations among the alleles A1-2III to A1-2V of the beta subclade were one and two differences (PGD 0.0011-0.0022) (Figs. 2b and $3 \mathrm{~b}$ ). The variation between the alleles A1-2I and A12II was eight differences (PGD 0.0087).

A similar mutation rate was indicated for the coding and the promoter region, because the variations were similarly distributed. This could be evidence that none of the detected Glu-A1-2 alleles code for a functional protein, as was shown for T. aestivum cv Cheyenne (A1-2I) and T. aestivum 'Chinese Spring' (A1-2II) (Shewry et al. 1999).

Distribution of alleles related to geographic origin and taxonomy

The frequencies of Glu-B1-1 and Glu-A1-2 alleles were calculated for wheat from different geographic origin or taxonomic units. 


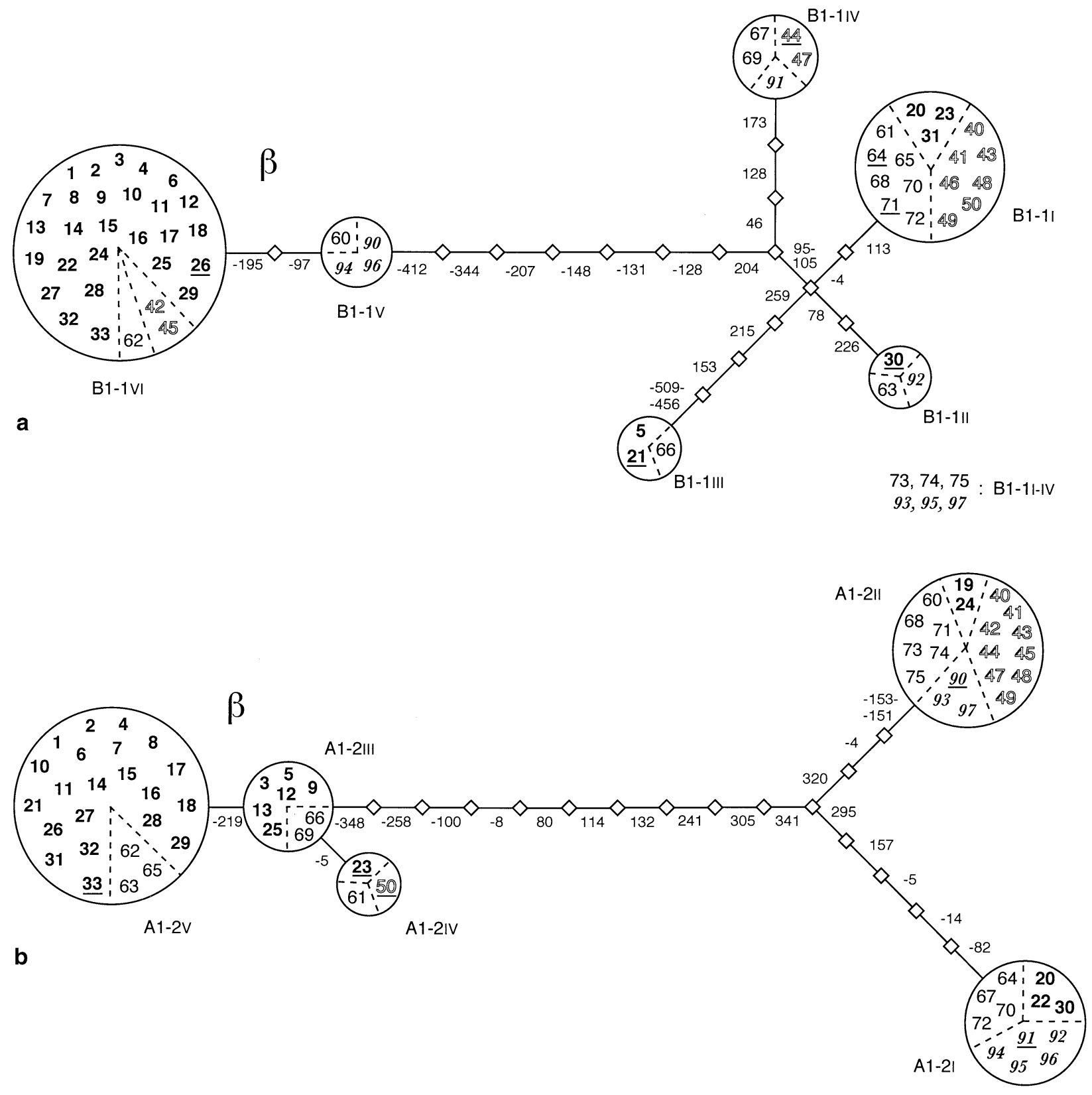

Fig. 3 Cladograms of six Glu-B1-1 alleles (a) and five Glu-A1-2 alleles (b) are shown. Each circle represents one identified allele. The size of the circles corresponds to the number of accessions with the respective allele. Different groups of wheat were indicated by different fonts of the lab numbers of each accession (table ESM): 1-33 (bold): European spelt, including the subgroup 1-19 consisting of spelt from the Northern Alpine region; 40-50 (contour): Asian spelt; 60-75 (normal): bread wheat; 90-97 (Baskerville,

In European spelt, beta alleles of Glu-B1-1 and GluA1-2 were most common (0.82 and 0.85 , respectively) and occurred in even higher frequencies (0.95 and 0.95) in spelt from the northern Alpine region (Table 1 and 2, Fig. 3). In contrast, the frequencies of Glu-B1-1 (0.19) and Glu-A1-2 (0.10) beta alleles were significantly lower in Asian spelt $(p<0.001$; chi-square test). Only the alleles B1-1VI and A1-2II were found in both European and italics): tetraploid wheat. For easier visualization wheats of different groups were additionaly separated by dotted lines within the circles. Beta subclades are marked with ' $\beta$ '. Six Glu-B1-1 sequences from the database could only be assigned to a range of alleles (B1-1I-IV). Empty nodes, where no alleles were observed, are symbolised by ' $\diamond$ '. The numbers between the nodes indicate the position of the informative sites according to Fig. 2. Sequences analysed additionally by direct sequencing are underlined

Asian spelt. The different geographic distribution of alleles corroborates an independent origin of European and Asian spelt as indicated in previous studies (Jaaska 1978; Dvorák et al. 1998; Dvorák and Luo 2001).

In free-threshing $T$. aestivum $(\mathrm{n}=16)$ the frequency of Glu-B1-1 beta alleles (0.13) was similar to Asian spelt. This suggests a closer relation of bread wheat to Asian spelt than to European spelt and is in agreement with 
Table 1 Frequencies of Glu-B1-1 alleles in different wheats. T. spelta Europe includes T. spelta from Switzerland and Germany ('N-Alps'). The frequencies of beta alleles are summarised

\begin{tabular}{llllllllll}
\hline Glu-B1-1 alleles & & B1-1I & B1-1II & B1-1III & B1-1IV & B1-1I-IV & B1-1V & $\begin{array}{c}\text { B1-1VI } \\
\text { 'Beta' alleles } \\
\text { (B1-1V-VI) }\end{array}$ \\
\hline T. spelta Europe & $(33)$ & 0.09 & 0.03 & 0.06 & 0.0 & - & 0.0 & 0.82 & 0.82 \\
T. spelta 'N-Alps' & $(19)$ & 0.0 & 0.0 & 0.05 & 0.0 & - & 0.0 & 0.95 & 0.95 \\
T. spelta Asia & $(11)$ & 0.64 & 0.0 & 0.0 & 0.18 & - & 0.0 & 0.18 & 0.18 \\
T. aestivum & $(16)$ & 0.38 & 0.06 & 0.06 & 0.19 & 0.19 & 0.06 & 0.06 & 0.12 \\
Tetraploid wheat & $(8)$ & 0.0 & 0.13 & 0.0 & 0.13 & 0.37 & 0.37 & 0.0 & 0.37 \\
\hline
\end{tabular}

${ }^{a}$ Six 240-bp sequences from the database could only be assigned to the group of alleles B1-1I to B1-1IV

Table 2 Frequencies of GluA1-2 alleles in different wheats. T. spelta Europe includes $T$. spelta from Switzerland and Germany ('N-Alps'). The frequencies of beta alleles are summarised

\begin{tabular}{llllllll}
\hline Glu-A1-2 alleles & & A1-2I & A1-2II & A1-2III & A1-2IV & A1-2V & $\begin{array}{l}\text { 'Beta' alleles } \\
\text { (A1-2II-V) }\end{array}$ \\
\hline T. spelta Europe & $(33)$ & 0.09 & 0.06 & 0.18 & 0.03 & 0.64 & 0.85 \\
T. spelta $N-A l p s '$ & $(19)$ & 0.0 & 0.05 & 0.26 & 0.0 & 0.69 & 0.95 \\
T. spelta Asia & $(10)$ & 0.0 & 0.90 & 0.0 & 0.10 & 0.0 & 0.10 \\
T. aestivum & $(16)$ & 0.25 & 0.37 & 0.13 & 0.06 & 0.19 & 0.38 \\
Tetraploid wheat & $(8)$ & 0.63 & 0.37 & 0.0 & 0.0 & 0.0 & 0 \\
\hline
\end{tabular}

previous results from the D-genome (Dvorák et al. 1998). Also the frequencies of Glu-A1-2 beta alleles (0.37) indicated a significantly closer relation of bread wheat to Asian than to European spelt $(p<0.001)$.

In tetraploid wheat three out of eight accessions (0.37) contained the beta allele B1-1V. This allele B1-1V was only found in tetraploid wheat and in a landrace of bread wheat from Germany (no. 60 in Fig. 3a), but never in spelt. On the other hand, the common European spelt beta allele B1-1VI was not found in tetraploid wheat. Also, no Glu-A1-2 beta alleles were found in tetraploid wheat. However, only a small number of tetraploid wheat was analysed in this study.

The analysed accessions of European spelt, Asian spelt, and bread wheat represent different taxonomic varieties (see ESM), based on morphological features (Szabo and Hammer 1996) but none of the taxonomic varieties were characterised by a specific combination of glutenin alleles.

\section{Discussion}

Despite many studies, important aspects about the relations between hulled and free-threshing hexaploid wheat remain controversial. One reason is that besides the normal inbreeding, a considerable amount of outcrossing occurs in fields of, for example, bread wheat (Griffin 1987; Martin 1990), and that wheat of different ploidy levels can be interfertile (e.g., McFadden and Sears 1946; MacKey 1966; Ohtsuka 1998). This allows many scenarios for obtaining genetic polymorphism. To study the relation between European spelt and other hexaploid wheats we focused on the A- and B-genomes, which originate from tetraploid wheat with the genomes AABB, by analysing the HMW Glu-B1-1 and Glu-A1-2 genes in different hexaploid wheats.
Our results show polymorphism at the HMW Glu-B1-1 and Glu-A1-2 loci in hexaploid wheat, and a differentiation of HMW Glu-B1-1 and Glu-A1-2 alleles in European spelt compared to other hexaploid wheats. Since the evolution of hexaploid wheat ca 8000 9000 years ago (Nesbitt and Samuel 1996), an average of 0.04-0.07 mutations should have occurred theoretically in the analysed $1-\mathrm{kb}$ fragment of the non-functional GluA1-2 genes based on an estimated rate of $5.1-7.7 \times 10^{-9}$ substitutions per site per year for introns and synonymous mutations in wheat (Allaby et al. 1999; Huang et al. 2002). Thus, the eight or more mutations, that separate the alleles A1-2I, A1-2II and the alleles A1-2III-V, can not have occurred since the evolution of hexaploid wheat. The five Glu-A1-2 alleles originate therefore from at least three, possibly five, different ancestors. Assuming a slower mutation rate for the functional Glu-B1-1 genes, the six Glu-B1-1 alleles in hexaploid wheat indicate a polyphyletic origin involving even six different ancestors. All Glu-B1-1 and Glu-A1-2 alleles, except for the alleles A1-2III-V, are separated from the closest related allele by at least two mutations. This lack of intermediate alleles is additional evidence that the mutations did not evolve continuously in hexaploid wheat but originate from different progenitors.

The polyphyletic origin of the A- and B-genomes in hexaploid wheat can be explained by independent hybridisations of tetraploid wheat and Ae. squarrosa, or by an introgression of tetraploid wheat into hexaploid wheat.

In the first scenario the hexaploid offspring is expected to differ from the other hexaploid wheat by independent A-, B- and D-genomes. In the second scenario only the Aand B-genomes of the speltoid alloploid would differ from its hexaploid ancestor, but not the D-genome. If the new alloploid of either scenario interbreeds subsequently with other hexaploid wheat, the genetic differentiation, i.e., the differences between hexaploid wheat species, 
would decrease. Nevertheless, allelic polymorphism, i.e., genetic variation within the hexaploid wheat species, would still indicate the polyphyletic origin. Comparing the variation and differentiation in the A- and B-genomes and in the D-genome can therefore help to distinguish alternative scenarios of spelt evolution.

Polymorphic loci on the D-genome show that hexaploid wheat evolved several times by hybridisations of tetraploid wheat and Ae. squarrosa (Dvorák et al. 1998; Talbert et al. 1998). Hybridisation and recombination events subsequently reduced differentiation and formed a common D-genome genepool of European spelt, Asian spelt and bread wheat (Dvorák et al. 1998). Several hybridisations of tetraploid wheat and Ae. squarrosa can thus explain allelic variation on the A-, B- and Dgenomes of hexaploid wheat, but not the differentiation of the A- and B-genomes, indicated for example by the glutenin genes in this study. The conjoint predominance of Glu-B1-1 and Glu-A1-2 beta alleles in European spelt, and the clear separation of beta alleles from non-beta alleles in both genes, can be best explained by an introgression of a tetraploid wheat carrying the beta alleles into hexaploid wheat. This introgression must have happened after the events reducing the differentiation of the D-genome genepool of hexaploid wheat so that the differentiation of the A- and B-genomes of hexaploid wheat was not affected. A secondary evolution of European spelt after bread wheat would also be in agreement with the archaeological record where spelt occurs after bread wheat in Europe (Maier 1996; Jacomet 1998). Yet, the origin of European spelt may not necessarily be in Europe.

Since tetraploid wheat with the genome AABB must have played an important role in any scenario of spelt evolution, the identification of the beta alleles of spelt in a tetraploid wheat could indicate where, and from which ancestors, European spelt originated. So far, however, no correlation was reported between the alleles of European spelt and tetraploid wheat for the A-genome and only little correlation for the B-genome. A marker on the Agenome, which was predominant in European spelt, was almost completely absent from cultivated tetraploid wheat (Dvorák and Luo 2001). Similarly, no Glu-A1-2 beta alleles were identified in the eight tetraploid wheat accessions that were available for this study. On the Bgenome, a spelt-specific gamma gliadin allele was closely related but not identical to an allele in tetraploid wheat (Von Büren 2001). This is similar to our results where the beta allele B1-1V in tetraploid wheat differed from the beta allele B1-1VI in European spelt by two mutations. Thus, it is not yet conclusive that European spelt acquired the beta alleles from a single ancestral tetraploid wheat.

Spelt and bread wheat accessions with either a Glu-B11 or a Glu-A1-2 beta allele show that in different hybridisation events only one beta allele was propagated. This could have happened through hybridisations of spelt and bread wheat as indicated by several non-beta alleles in European spelt, or by an introgression of tetraploid wheat with a single beta allele into bread wheat as indicated by the beta allele B1-1V of tetraploid wheat in a bread wheat accession (no. 60).

The higher genetic variation of the Glu-B1-1 and GluA1-2 alleles in bread wheat compared to European and Asian spelt can be explained by the much wider geographic range of bread wheat cultivation, which faciliates higher geneflow due to natural out-crossing and human selection and trade. In turn, the little allelic variation at the Glu-B1-1 and Glu-A1-2 loci in European spelt, particularly from the Northern Alpine region, can be explained by the geographically restricted growing area and a secondary origin. In Asian spelt, low allelic variation can be similarly explained. In contrast to European spelt, however, the limited differentiation of the glutenin alleles between Asian spelt and bread wheat would allow a scenario where Asian spelt originates from the hulled ancestors of bread wheat.

Although many of the analysed accessions were landraces, which are expected to be less affected by modern wheat breading than modern cultivars, it is difficult to determine how much of the genetic variation, especially in bread wheat and in European spelt, is ancient, and how much is due to recent events. Data from pre-industrial times come from a historical spelt (accession number 9) and bread wheat sample (no. 75) (Blatter et al. 2002). They show that the Glu-B1-1 and Glu-A1-2 beta alleles, which are predominant in European spelt, existed at least 300 years ago.

Acknowledgements This work was funded by the Swiss National Science Foundation (grant no. 31-37'290.93), the 'Novartis foundation' and the 'Freie Akademische Gesellschaft' Basel.

\section{References}

Allaby RG, Banerjee M, Brown TA (1999) Evolution of the highmolecular-weight glutenin loci of the A, B, D and G genomes of wheat. Genome 42:296-307

Anderson OD, Greene FC (1989) The characterization and comparative analysis of high-molecular-weight glutenin genes from genomes A and B of a hexaploid bread wheat. Theor Appl Genet 77:689-700

Andrews AC (1964) The genetic origin of spelt and related wheats. Züchter 34:17-22

Blatter RHE, Jacomet S, Schlumbaum A (2002) Spelt-specific alleles in HMW glutenin genes from modern and historical European spelt (Triticum spelta L.). Theor Appl Genet 104:329-337

Brown TA (1999) How ancient DNA may help in understanding the origin and spread of agriculture. Phil Trans R Soc Lond B 354:89-98

Clement M Posada D, Crandall KA (2000) TCS: a computer program to estimate gene genealogies. Mol Ecol 9:1657-1660

Dvorák J, Luo M-C (2001) Evolution of free-threshing and hulled forms of Triticum aestivum: old problems and new tools. In: Caligari PDS, Brandham PE (eds) The Linnean, Special Issue No 3. Wheat Taxonomy: the legacy of John Percival, Academic Press, London, pp 127-136

Dvorák J, Luo M-C, Yang Z-L, Zhang H-B (1998) The structure of the Aegilops tauschii genepool and the evolution of hexaploid wheat. Theor Appl Genet 97:657-670

Flaksberger C (1930) Ursprungszentrum und geographische Verbreitung des Spelzes (Triticum spelta L.). Angew Bot 12:86-99 
Griffin WB (1987) Outcrossing in New Zealand wheats measured by occurrence of purple grain. NZ J Agric Res 30:287-290

Huang S, Sirikhachornkit A, Su X, Faris J, Gill B, Haselkorn R, Gornicki P (2002) Genes encoding plastid acetyl-CoA carboxylase and 3-phosphoglycerate kinase of the Triticum/Aegilops complex and the evolutionary history of polyploid wheat. Proc Natl Acad Sci USA 99:8133-8

Jaaska V (1978) NADP-dependent aromatic alcohol dehydrogenase in polyploid wheats. Theor Appl Genet 53:209-217

Jaaska V (1980) Electrophoretic survey of seedling esterases in wheat in relation to their phylogeny. Theor Appl Genet 56:273284

Jacomet S (1998) Einige weitere Schlüsse aus den bronzezeitlichen Pflanzenspektren. In: Hochuli S, Niffeler U, Rychner V (eds) SPM III: Bronzezeit, Schweizerische Gesellschaft für Ur- und Frühgeschichte. Basel, pp 167-170

Kerber ER, Rowland GG (1974) Origin of the free-threshing character in hexaploid wheat. Can J Gen Cytol 16:145-154

Kihara H (1944) Discovery of the DD-analyser, one of the ancestors of Triticum vulgare. Agric Hort 19:13-14

Kuckuck H, Schiemann E (1957) Über das Vorkommen von Spelz und Emmer (Triticum spelta L. und T. dicoccum Schübl.) im Iran. Zeit Pflanzenzüchtg 38:383-396

Liu YG, Tsunewaki K (1991) Restriction fragment length polymorphism (RFLP) analysis in wheat. II. Linkage maps of the RFLP sites in common wheat. Jpn J Genet 5:617-33

MacKey J (1966) Species relationship in Triticum. In: MacKey J (ed) Proc Int Wheat Genet Symp, Hereditas (Suppl) 2:237-276

Maier U (1996) Morphological studies of free-threshing wheat ears from a Neolithic site in Southwest Germany, and the history of the naked wheats. Veg Hist Archaeobot 5:39-55

McFadden ES, Sears ER (1946) The origin of Triticum spelta and its free-threshing hexaploid relatives. J Hered 37:81-89

McIntosh RA, Hart GE, Devon KM, Gale MD, Rogers WJ (1998) Catalogue of gene symbols for wheat. In: Slinkard AE (ed) Proc 9th Int Wheat Genet Symp, vol 5. Extension Press, University of Saskatchewan, Saskatoon, Canada, pp 1-235
Martin TJ (1990) Outcrossing rates in 12 hard red winter wheat cultivars. Crop Sci 30:59-62

Miller TE (1987) Systematics and evolution. In: Lupton FGH (ed) Wheat breeding. Chapman and Hall, London New York, pp 130

Nesbitt M, Samuel D (1996) From staple crop to extinction? The archaeology and history of the hulled wheats. In: Padulosi S, Hammer K, Heller J, Pacoli C (eds) Hulled wheats. Int Plant Genet Res Inst, Rome, Italy, pp 41-100

Ohtsuka I (1998) Origin of central European wheat. In: Slinkard AE (ed) 9th Int Wheat Genet Symp, University of Saskatchewan ExtensionPress, Saskatoon pp 303-305

Rozas J, Rozas R (1999) DnaSP version 3: an integrated program for molecular population genetics and molecular evolution analysis. Bioinformatics 15:174-175

Salamini F, Özkan H, Brandolini A, Schäfer-Pregl R, Martin W (2002) Genetics and geography of wild cereal domestication in the Near East. Nature Rev Genet 3:429-441

Shewry PR, Tatham AS, Halford NG (1999) The prolamins of the Triticeae. In: Shewry PR, Casey R (eds) Seed proteins. Kluwer Academic Publisher, The Netherlands, pp 35-78

Siedler H, Messmer MM, Schachermayr GM, Winzeler H, Keller B (1994) Genetic diversity in European wheat and spelt breeding material based on RFLP data. Theor Appl Genet 88:994-1003

Szabo AT, Hammer K (1996) Notes on the taxonomy of farro: Triticum monococcum, $T$. dicoccon and $T$. spelta. In: Padulosi S, Hammer K, Heller J, Pacoli C (eds) Hulled wheats. Int Plant Genet Res Inst, Rome, Italy, pp 41-100

Talbert LE, Smith LY, Blake NK (1998) More than one origin of hexaploid wheat is indicated by sequence comparison of lowcopy DNA. Genome 41:402-407

Von Büren M (2001) Polymorphisms in two homeologous gammagliadin genes and the evolution of cultivated wheat. Genet Res Crop Evol 48:205-220

Zohary D, Hopf M (2000) Domestication of plants in the old world, 3rd edn. Oxford University Press, Oxford, UK 